\title{
Necrotizing lymphadenitis may be induced by overexpression of Toll-like receptor7 (TLR7) caused by reduced TLR9 transport in plasmacytoid dendritic cells (PDCs)
}

\author{
Shigeyuki Asano, ${ }^{1)}$ Hiroko Sato, ${ }^{2)}$ Kikuo Mori, ${ }^{1)}$ Kazuki Yamazaki, ${ }^{1)}$ Hiroyuki Naito, ${ }^{2)}$ \\ Hoshiro Suzuki ${ }^{3)}$
}

\begin{abstract}
Necrotizing lymphadenitis (NEL) is a self-limited systemic disease exhibiting characteristic clinical features. The pathogenesis of the disease remains unclear, but it may be associated with viral infection. In lymph nodes affected by this disease, innumerable plasmacytoid dendritic cells produce interferon- $\alpha$ when triggered by certain viral stimuli. IFN- $\alpha$ presents antigens causing the transformation of $\mathrm{CD}^{+}$cells into immunoblasts and apoptosis of $\mathrm{CD}^{+}$cells. From the perspective of innate immunity, UNC93B1, an endoplasmic reticulum (ER)-resident protein, associates more strongly with TLR9 than TLR7. Homeostasis is maintained under normal conditions. However, in NEL, TLR 7 was observed more than TLR 9, possibly because mutant type UNC93B1 associates more tightly with TLR7. The inhibitory effects against TLR7 by TLR9 were reported to disappear. It is likely that more TLR7 than TLR9 is transported from the ER to endolysosomes. In conclusion, overexpression of TLR7, an innate immune sensor of microbial single-stranded RNA, is inferred. Consequently, NEL may be induced.
\end{abstract}

Keywords: necrotizing lymphadenitis; plasmacytoid dendritic cells; TLR7; TLR9; UNC93B1

\section{INTRODUCTION}

The pathogenesis of necrotizing lymphadenitis (NEL), a systemic reactive disease characterized by blastic transformation of $\mathrm{CD} 8^{+}$cells and apoptosis of $\mathrm{CD} 4^{+}$cells, remains unclear. However, it may be related to viral infection, as inferred from clinicopathological findings. ${ }^{1-8}$ With NEL, innumerable plasmacytoid dendritic cells (PDCs) are observed in lymph nodes. The PDCs produce large quantities of interferon- $\alpha($ IFN- $\alpha)$. In addition to antiviral reaction by IFN- $\alpha$ production, PDCs play a role in antigen presentation, tolerance, and cytotoxic function. Although these functions described above are recognized as innate-immunity mediated by Toll-like receptor7 (TLR7) and TLR9 in the PDCs, no study has examined the relationship between NEL and innate immunity. We explain the roles of TLR7, TLR9, UNQ93B1, and MX1 in the PDCs of NEL as an approach to elucidating the etiology of NEL.

\section{MATERIALS AND METHODS}

Data of 70 cases of NEL during 1989-2016 were collected at Iwaki Kyoritsu General Hospital in Japan. All patient files kept at the hospital were used for this study.

Lymph node biopsy samples were divided into three: one was fixed in $10 \%$ formalin for routine diagnosis, the second was fixed in $2.5 \%$-glutaraldehyde for conventional electron microscopy (EM), and the third was used for imprint cytology, being fixed in $95 \%$ ethyl alcohol. Sections cut from $10 \%$-formalin-fixed paraffin-embedded (FFPE) lymph node samples were stained with hematoxylin-eosin (HE), Giemsa stain, periodic acid-Schiff (PAS), silver impregnation, and elastica-Masson stain. Imprint cytological specimens on slide glasses were stained with Papanicolaou and Giemsa stains. For immunohistochemical analysis (IHC), FFPE tissue sections ( $4 \mu \mathrm{m}$ thickness) were deparaffinized in xylene, dehydrated through graded alcohols, and rinsed in distilled water. Antigen retrieval was performed in medium using a pressure cooker (Panasonic Co. Ltd., Tokyo, Japan) for 20

\footnotetext{
Received: November 14, 2020. Revised: January 23, 2021. Accepted: February 15, 2021. J-STAGE Advance Published: May 14, 2021 DOI: $10.3960 /$ jslrt.20060

${ }^{1}$ Pathology Center, Iwaki City Medical Center, Iwaki, Japan, ${ }^{2}$ Department of Dentistry and Oral Surgery, Iwaki City Medical Center, Iwaki, Japan, ${ }^{3}$ Department of Pediatrics, Iwaki City Medical Center, Iwaki, Japan

Corresponding author: Shigeyuki Asano, Pathology Center, Iwaki City Medical Center, Iwaki, Fukushima, 973-8555, Japan. E-mail: patho.asano@gmail.com Copyright (C) 2021 The Japanese Society for Lymphoreticular Tissue Research

(c) BY-NC-SA This work is licensed under a Creative Commons Attribution-NonCommercial-ShareAlike 4.0 International License.
} 
min. IHC was conducted using an automated stainer (Nichirei Biosciences Inc., Tokyo, Japan) according to the manufacturer's instructions. Regarding the dilution ratio of each antibody, it was diluted from the highest ratio specified in the instructions, and the dilution concentration was adjusted until the immune response of the tissue was optimized. In this study, it was diluted in antibody diluent (Dako, Inc., Tokyo, Japan). As positive controls, a lymph node sample of NEL was used; CD123 antibody marked PDCs, Mx1 antibody marked interferon-producing cells, and TLR7, TLR9, and UNQ93B1 antibodies were used to mark organelles in PDC. Correct responses were judged to be positive. The final optimal dilution ratio of each antibody is shown in Table 1. The negative control was confirmed by staining only with diluent. As the activator, $\mathrm{pH} 9$ citrate buffer was used for CD123, TLR7, and TLR9, pH6 citrate buffer was used for UNC93B1, and none was used for Mx1, as described in the manual. The sections were mounted with Malinol (Muto Pure Chemicals Co. Ltd., Tokyo, Japan). The panel of antibodies is shown in Table 1.

In five lesions and normal regions of the same tissue sections of 23 different temporal cases, 400 cells in each were counted using an attached meshed eyepiece. The percentages of $\mathrm{CD} 123^{+}, \mathrm{TLR}^{+}$, TLR9, $\mathrm{UNC} 93 \mathrm{~B} 1^{+}$, and $\mathrm{Mx} 1^{+}$cells were recorded.

\section{RESULTS}

\section{Clinical findings}

Clinical features of the patients are presented in Figures 1a-1d. We examined 24 male and 46 female patients $(\mathrm{M} / \mathrm{F}=$ $1 / 1.92$ ) who were 2-55 years of age (average, 25.8 years; median, 26.0 years; $71.4 \%$ younger than 30 years). The peak of onset was in the 20s (25.7\%) (Fig. 1a). No seasonal occurrence was found. Most patients complained of dull or acutely painfully swollen cervical (80.9\%) lymph nodes (Fig. $1 b)$, most frequently on the left side. In addition, initial tonsillar swelling, transient skin rash, and hepatosplenomegaly were observed. A skin rash similar to rubella or druginduced eruption was common in febrile and severe cases. Fever seldom responded to antibiotics, but steroids and antichloristics were effective. The period of illness was almost invariably a single attack, although five cases $(1.3 \%)$ recurred 2-10 years later. In cases with available peripheral white blood cell counts, $46.6 \%(27 / 58)$ of patients had a count lower than $4,000 / \mathrm{mm}^{3}$ (Fig. 1c). In peripheral blood, CD ${ }^{+}$ cells were more abundant than $\mathrm{CD}^{+}$cells from the onset of the disease, but $\mathrm{CD} 8^{+}$cells decreased gradually through the clinical course, producing an increasing $\mathrm{CD} 4^{+} / \mathrm{CD} 8^{+}$cell ratio (Fig. 1d).

\section{Pathological findings}

Surgical biopsies were performed 4 to 161 days after onset. Lymph nodes, which were smooth and soft, had diameters as large as $3 \mathrm{~cm}$. Irregular yellowish foci were often observed on the cut surface (Fig. 2a). Several cases exhibited periadenitis with capsular thickening. Lesions appeared mainly in the paracortical area, where the lymphocyte depletion was greatest. Lymph follicles remained sporadically in the lesion. Histological features of involved lymph nodes included the presence of immunoblasts, PDCs, histiocytes, and macrophages, the latter with phagocytized nuclear debris originating mainly from apoptotic lymphocytes (Fig. 2b-c). PDCs were distributed more irregularly in the immunoblastic predominant peripheral area than in the central necrotic and degenerated area. The number of PDCs in lymph node lesions increased gradually with time. The PDC morphology was slightly larger and more irregular in the lesions than in the non-lesion areas of lymph nodes (Fig. 2d). On EM, PDCs had linear chromatin located on the cell membrane. Often, nucleoli were observed. Many lamellar structures and long rough endoplasmic reticulum (rER), main cytoplasmic organelles, were aggregated on one side of the cytoplasm (Fig. 2e). They have no phagocytotic function, but tubuloreticular structures (TRS) were sometimes observed in cytoplasm. Numerous immunoblasts were located at the periphery rather than in the center of the lesions. No granulocytes or bacteria were found. The cell composition varied from case to case and over time. Xanthomatous foamy macrophages were noted in some cases.

$\mathrm{TLR}^{+}$cells increased over time and distributed more in peripheral areas than in the central lesions, which coincides with the distribution of PDCs (Fig. 2f). Although TLR7 ${ }^{+}$ cells localized to part of the cytoplasm in PDCs, there were few TLR9 ${ }^{+}$cells (Fig. 2g). Furthermore, they were scattered in the peripheral area of the lesions. Many $\mathrm{UNC} 93 \mathrm{~B} 1^{+}$cells were found in the lesion area, mainly in the vicinity of $\mathrm{HEV}$, and also scattered between lymphocytes in the non-lesion area. UNC $93 \mathrm{B1}^{+}$cells were consistent with PDCs (Fig.

Table 1. Table 1: Antibodies used for the immunohistochemical examination.

\begin{tabular}{llllll}
\hline Antibody & Clone & & Source & Clonality & Retrieval \\
\hline CD123 & $6 \mathrm{H} 6$ & Dendritic cells, myeloid precursor cells, macrophages & Bio & M & PC $(1: 100)$ \\
Mx1 & IFI-78K & Interferon-induced GPT-binding protein Mx1(MxA) & Therm & P & PC $(1: 1,000)$ \\
TLR7 & $66 \mathrm{H} 3$ & Dendritic cells & Novus & M & PC $(1: 50)$ \\
TLR9 & $5 \mathrm{G} 5$ & PDCs, monocytes & Abcam & M & PC (1:10) \\
UNC93B1 & - & Muscle contraction & Abcam & P & PC $(1: 400)$ \\
\hline
\end{tabular}

Bio, BioLegend, CA, USA; Therm, Thermo Scientific, IL, USA; Novus, Novus Biologicals, CO, USA; Abcam, Abcam Co. Ltd., MA, USA; Bioss, Bioss Co. Ltd., Cambridge, UK; $M$, monoclonal; P, polyclonal; PC, pressure cooker; Non, non-treated 


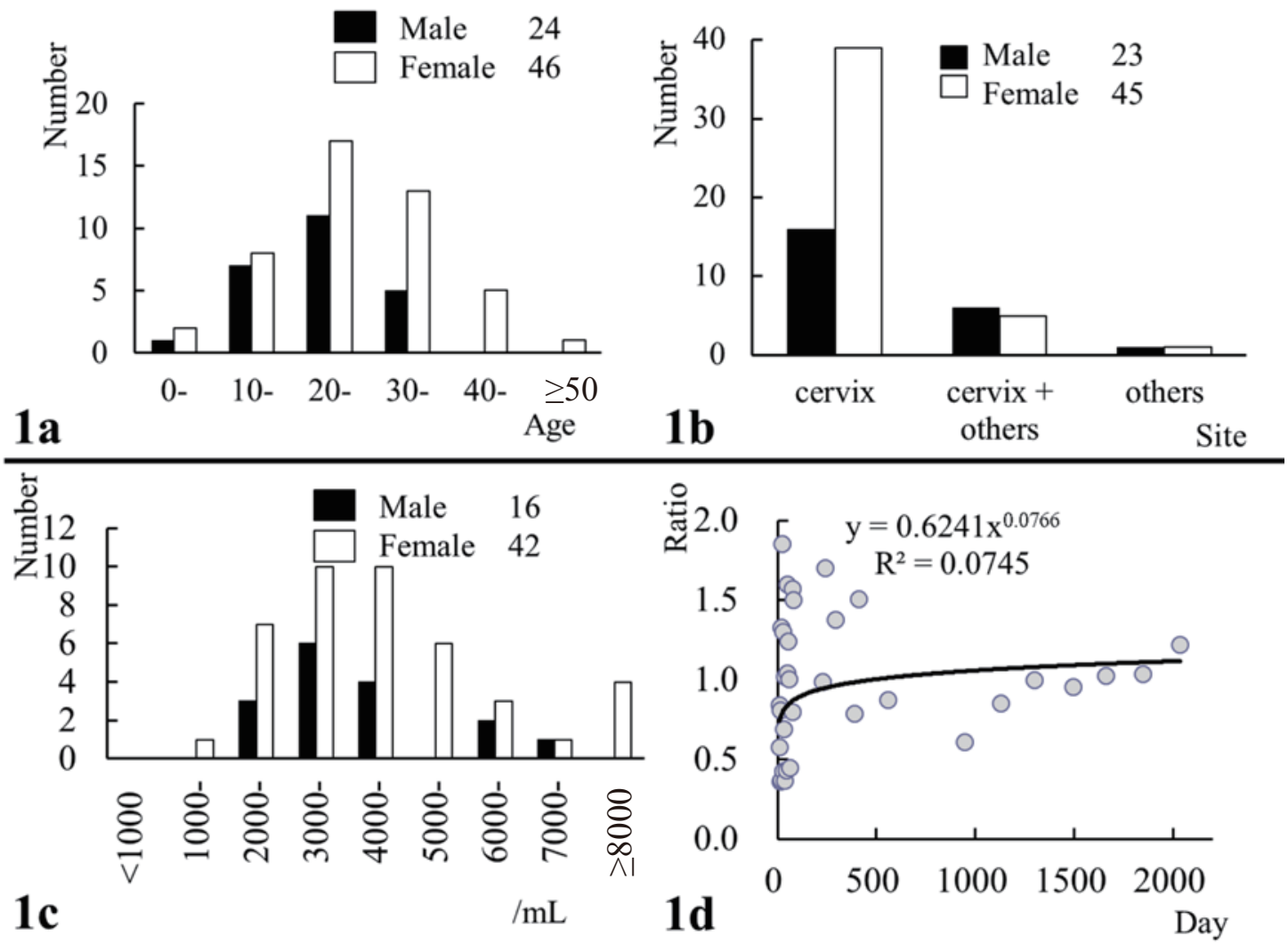

Fig. 1. Clinical findings. (a) Age and sex distribution of 70 cases. Most patients were younger than 30 years old $(71.4 \%, 50 / 70)$. (b) Site of affected lymph nodes in 66 cases. Cervical lymph nodes were the most common affected site. (c) White blood cells in peripheral blood in 58 cases. The white blood cell count is less than $4,000 / \mathrm{mm}^{3}$ $(46.6 \%, 27 / 58)$. (d) CD4+/CD $8+$ cell ratio of the peripheral blood in 8 cases (34 samples). The ratio is lower than 1.0 in the early stage, but it gradually approaches 1.0.

2h). $\quad \mathrm{Mx}^{+}$cells included PDCs, macrophages, immunoblasts, and endothelial cells, but no small lymphocytes (Fig. 2i). The numbers of $\mathrm{CD} 123^{+}, \mathrm{TLR}^{+}, \mathrm{UNC} 93 \mathrm{~B} 1^{+}$, and $\mathrm{Mx} 1^{+}$ cells in affected lesions increased over time, but those of $\mathrm{TLR}^{+}$cells did not change. In non-affected lesions, the numbers of these cells were mostly unaltered (Fig. 3a-b).

\section{DISCUSSION}

NEL is a systemic reactive disease with characteristic clinical features, such as high fever, painful cervical lymphadenopathy, and leukopenia, without seasonal occurrence. Although the pathogenesis of the disease remains unclear, it may be associated with viral infection, as inferred from the following: antibiotics are ineffective, ${ }^{1,2}$ no granulocytes are found in lymph node lesions, ${ }^{3,4}$ tubular inclusions, such as TRS and parallel tubular array (PTA), are frequently observed; ${ }^{1,2,5}$ and increased $2^{\prime}-5^{\prime}$ oligoadenylic acid-synthesizing enzyme ${ }^{6}$ and IFN- $\alpha$ induces Mx1 protein expression in lymph node lesions. ${ }^{7}$ In addition, $\mathrm{CD} 8^{+}$cells transform into immunoblasts and $\mathrm{CD} 4{ }^{+}$cells undergo apoptosis. ${ }^{8}$ Macrophages and PDCs play an important role in the immune response of NEL patients. ${ }^{9,10}$

In NEL, innumerable PDCs, consistent with $\mathrm{Mx} 1^{+}$cells, surrogate markers for IFN- $\alpha$, are observed not only in intact areas, but also in lesions, including those in and around high endothelial venules (HEV) of lymph nodes. ${ }^{7}$ Indeed, PDCs are characteristic cells. Their morphology resembles that of immunoglobulin-secreting plasma cells. They can be uniquely identified in blood and affected lymph nodes of NEL by their high levels of interleukin- 3 receptor $\alpha$ chain (CD123) combined with other cell markers such as CD45RA, CD68, and HLA-DR. ${ }^{7,11-15}$ PDCs are continuously produced from bone marrow. After leaving the bone marrow, they migrate into T-cell-rich areas of the lymphoid tissues through HEV in lymph nodes. ${ }^{16}$ The PDC morphology is slightly larger and more irregular in lesions of lymph nodes, ${ }^{7}$ which may reflect their maturation. Moreover, they produce large amounts of IFN- $\alpha$ when triggered by certain viruses and bacterial stimuli. ${ }^{12,17-20}$ They present antigens: $\mathrm{CD} 8^{+}$cells transform into immunoblasts and $\mathrm{CD} 4^{+}$cells undergo apoptosis. ${ }^{7,821-25}$ The density of these cells correlates well with the high number of $\mathrm{Mx} 1^{+}$cells, suggesting that PDCs produce IFN- $\alpha$ in lymph node lesions. ${ }^{7,26}$ Consequently, PDCs have important functions, such as innate immunity and adaptive immunity, as described above. $\mathrm{Mx} 1^{+}$cells are significantly more abundant than $\mathrm{TLR} 7^{+}$cells and $\mathrm{CD} 123^{+}$cells from the early stages of the disease, which suggests that the production of IFN- $\alpha$ for host defense is rapid and powerful against pathogens in this disease.

In Drosophila, Toll was first identified as a fundamentally important molecule for dorso-ventral patterning of the 

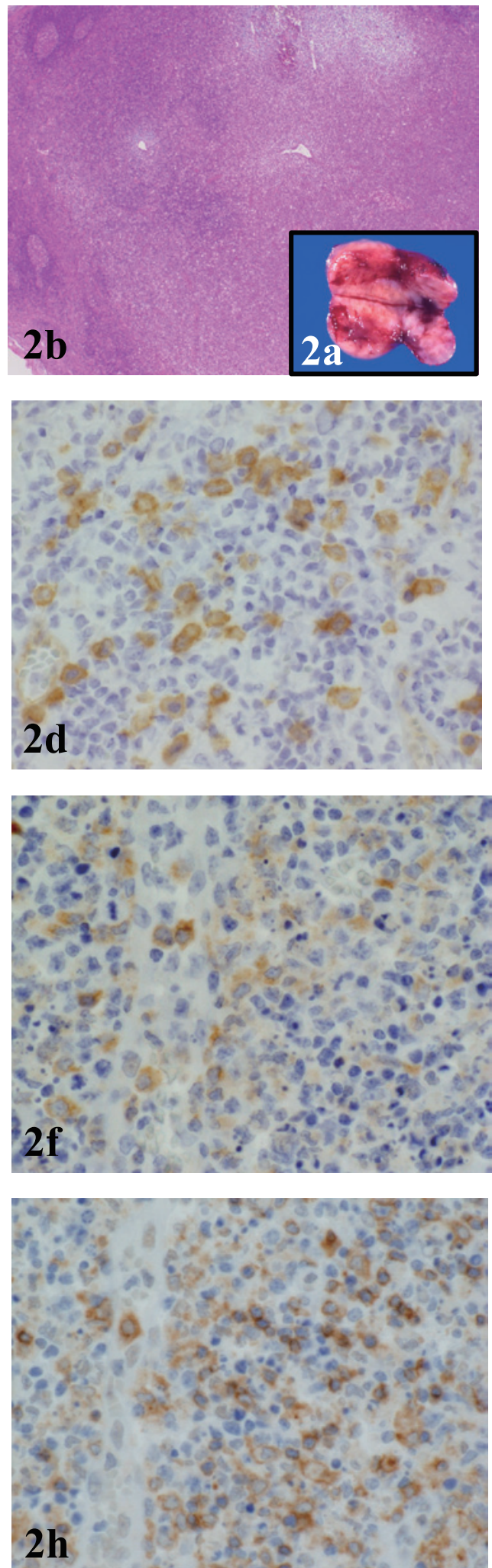
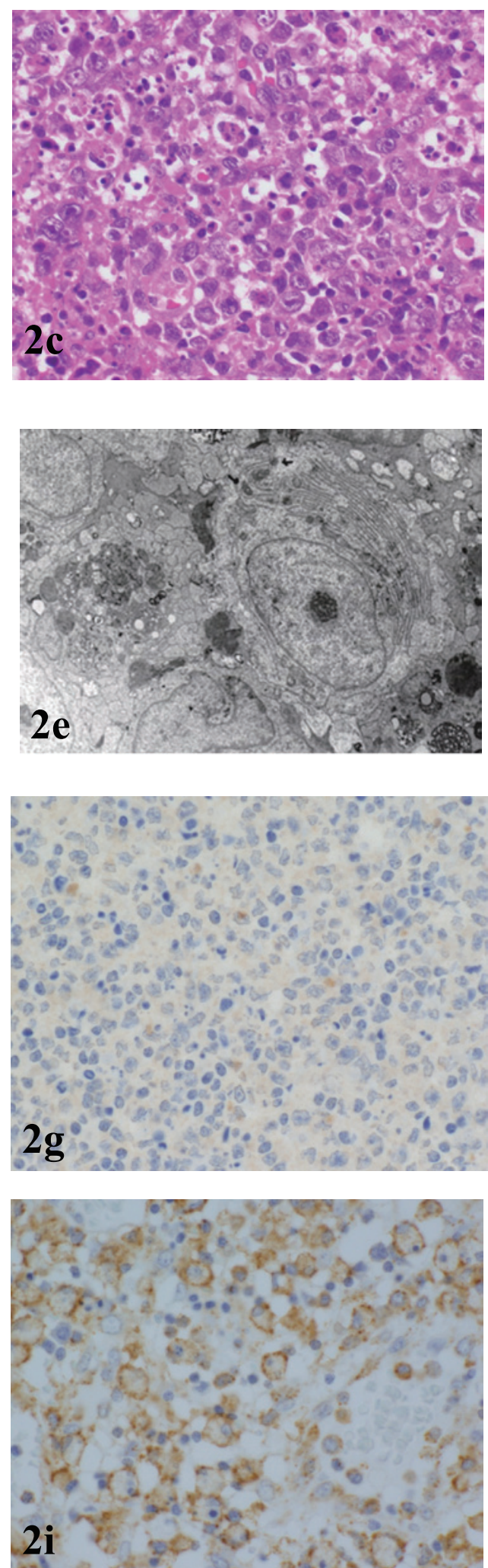

Fig. 2. Characteristic histological features of NEL: (a) Macroscopic cut surface of the affected lymph node shows soft and patchy hemorrhagic lesions. (b) Main lesions coincide with the pale area (HE). (c) Enlargement of $(\boldsymbol{b})$ shows many immunoblasts, histiocytes, PDCs, lymphocytes, and macrophages phagocytizing apoptotic lymphocytes. (d) CD $123^{+}$cells coincide with PDCs. They are distributed throughout the lesions. $(\boldsymbol{e})$ Electron microscopy of a PDC. PDCs have characteristic eccentrically distributed nuclei and lamellar rough ER in the cytoplasm. $(f)$ TLR7 $7^{+}$cells distribute throughout the lesions. Their distribution pattern is almost identical to that of PDCs. TLR7 localizes to a part of the cytoplasm around the nuclei of PDCs. $(\mathrm{g})$ There are fewer TLR $9^{+}$cells than TLR $7^{+}$ cells. The reaction is weak. (h) Many UNC93B $1^{+}$cells are found in the vicinity of the HEV of the lesion. These are consistent with large, PDCs with irregularly shaped nuclei. $(i) \mathrm{Mx} 1^{+}$cells included PDCs, macrophages, immunoblasts, and high endothelial cells among small lymphocytes throughout lesions and non-lesions. 


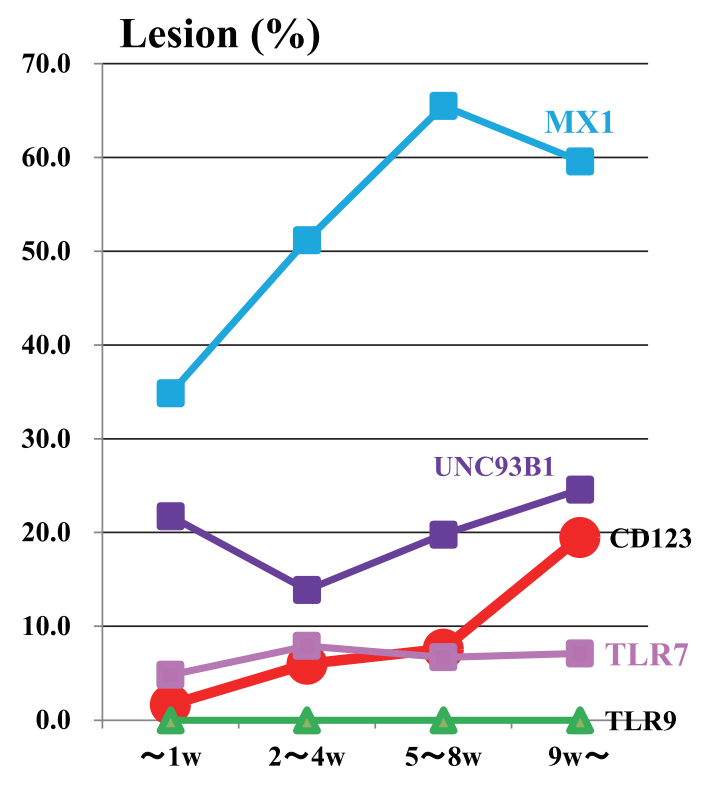

a

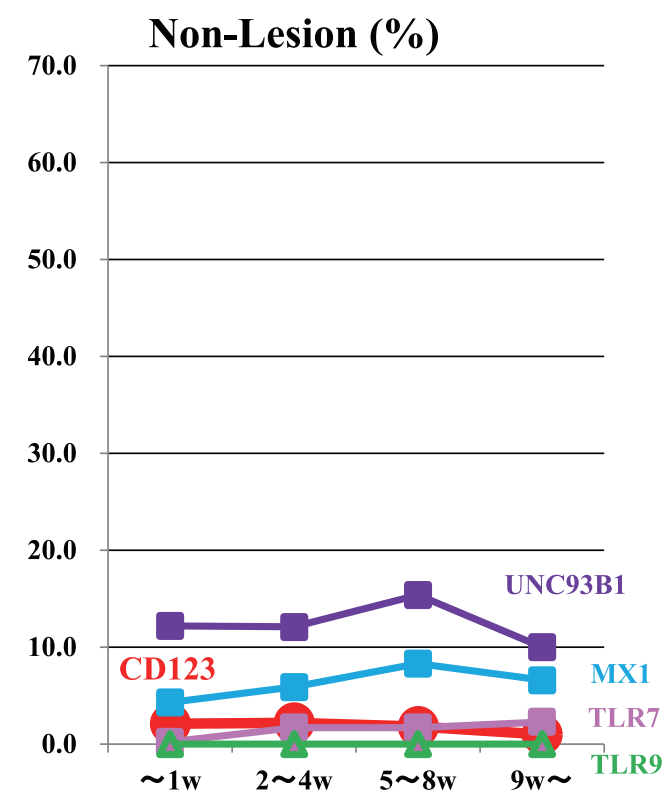

b

Fig. 3. Temporal changes in the proportions of CD123+, $\mathrm{TLR}^{+}, \mathrm{TLR}^{+}, \mathrm{UNQ} 93 \mathrm{~B} 1^{+}$, and $\mathrm{MX} 1^{+}$cells in the lymph node. (a) Lesions: The number of TLR7 ${ }^{+}, \mathrm{CD}_{123^{+}}$, and $\mathrm{UNC} 93 \mathrm{~B} 1^{+}$cells increase gradually with time, but the number of $\mathrm{Mx}^{+}$cells sharply increased from the early stages. The number of TLR9 ${ }^{+}$cells was unchanged. (b) Nonlesions: No changes were detected.

embryo, and subsequently as a key molecule for the antifungal immune response in adults. ${ }^{27,28}$ A homologous family of Toll receptors, termed Toll-like receptors (TLRs), exists in vertebrates, including humans. ${ }^{29-31}$

Ten types of TLRs have been found in humans. They are classified largely into two subfamilies based on their localization, cell surface TLRs and intracellular TLRs. Cell surface TLRs include TLR1, TLR2, TLR4, TLR5, TLR6, and TLR10, whereas intracellular TLRs are localized in the endosomes and endolysosomes, and include TLR3, TLR7, TLR8, and TLR9. ${ }^{32-34}$ Cell surface TLRs mainly recognize microbial membrane components such as lipids and proteins. TLR4 recognizes bacterial lipopolysaccharide. TLR2 along with TLR1 or TLR6 widely recognizes microbial pathogens, including lipoproteins and peptidoglycans, and mycoplasma lipopeptides. ${ }^{32}$ TLR10 can also sense influenza A virus infection. ${ }^{35}$ TLR10 collaborates with TLR2 to recognize ligands from listeria. ${ }^{36}$ TLR5 recognizes bacterial flagellin. ${ }^{27}$ Intracellular TLRs recognize nucleic acids from bacteria and viruses, and also recognizes self-nucleic acids in diseases such as autoimmunity. ${ }^{34}$ TLR3 recognizes viral doublestranded RNA (ds-RNA), small interfering RNA, and selfRNA from damaged cells. ${ }^{33,37-39}$ TLR7/8 is predominantly expressed in PDCs. It recognizes single-stranded RNA (ssRNA) from ss-RNA viruses such as vesicular stomatitis virus, influenza virus, or Newcastle disease virus. ${ }^{40-43}$ It also recognizes RNA from streptococcus B bacteria in conventional dendritic cells. ${ }^{44}$ TLR9 in PDCs recognizes bacterial and viral DNA that is rich in unmethylated cytidine-phosphate-guanosine DNA (CpG-DNA) motifs. ${ }^{45}$ Among the 10 TLRs described above, PDCs are a subset of DCs with the capacity to secrete vast amounts of IFN- $\alpha$ in response to viral infection via TLR7 and TLR9.

TLR7 and TLR9 are synthesized in the ER and migrate through the Golgi into endosomes and endolysosomes. Resting PDCs predominantly express TLR7 and TLR9, and reside in the ER in association with UNC93B1 and gp96. Within the ER in PDCs, chaperone proteins, such as gp96, ERCa2 ${ }^{+}$, PRAT4A, and UNC93B1, are required for TLR7 and TLR9 to exit the ER, although TLR7 and TLR9 compete for binding to UNC93B1. Under normal circumstances, the $\mathrm{N}$-terminal domain of UNC93B1 associates more strongly with TLR9, resulting in more robust signaling through TLR9 but weak TLR7 responses. Consequently, UNC93B1 controls the trafficking of intercellular TLR7 and TLR9 from the ER to endosomes. It regulates excessive TLR7 activation using TLR9 to counteract TLR7.

IHC analyses revealed the existence of many $\mathrm{UNC} 93 \mathrm{~B} 1^{+}$ cells in NEL, as described above. UNC93B1 can bind strongly to TLR7, but not to TLR9, in NEL. A UNC93B1 deletion mutant lacking this $\mathrm{N}$-terminal domain associates more tightly with TLR7 and less tightly with TLR9. Indeed, TLR7-dependent diseases, such as NEL, may be induced. ${ }^{34,47,48}$ Overexpression of TLR7, which represents an imbalance between TLR7 and TLR9, may engender disorders, such as NEL and systemic lupus erythematosus (SLE), and even the presence of TLR9. ${ }^{16,33,34,46}$ UNC93B1 is ultimately necessary for intracellular TLRs responses, as it regulates trafficking from the ER to the endolysosomes and responds to the ligands. ${ }^{34}$ TLR7 of PDCs in NEL may be a recognition sensor of ss-RNA from ss-RNA viruses. ${ }^{16,40-43}$

In $\mathrm{B}$ cells and PDCs of SLE, TLR7 is activated by 
RNA-containing immune complexes; TLR9 is activated by DNA-containing immune complexes. After sensing these TLRs, cytokine secretion and T cell activation by B cells and PDCs are observed. In addition, $\mathrm{B}$ cells are in charge of antigen production, whereas PDCs are in charge of IFN- $\alpha$ secretion. ${ }^{33,36}$ Signaling pathways of TLR7 and TLR9 in NEL and SLE are dependent on myeloid differentiation primary-response gene 88 (MyD88). The adaptor molecular complex (MyD88-TRAF6-IRAK4) then activates interferonregulatory factor 7 (IRF7) and propagates the downstream signals. Following ubiquitination and phosphorylation, IRF7 translocates to the nucleus and induces IFN- $\alpha$ production, which affects innate and adaptive immunity. The signal pathway described above explains IFN- $\alpha$ production excluding other cytokines (Fig. 4). ${ }^{34,49}$

Although IFN- $\alpha$ are induced via TLR7 and TLR9 in PDCs of NEL and SLE, there are different pathogens between these diseases such as nucleic pathogens (ss-RNA and CpG-DNA) in NEL and immune complexes in SLE. In SLE, high rates of cell death, lack of clearance, impaired phagocytosis, decreased or absent DNase, and TLR7 and TLR 9 imbalance are observed. ${ }^{3,33,34}$ In NEL, apoptotic cells, not necrotic cells, which are present in SLE, are rapidly phagocytized by macrophages. They do not release their intracellular contents. As a result, PDCs in NEL do not produce proinflammatory cytokines. In addition, they do not attract neutrophils, which is an important characteristic of NEL. ${ }^{50}$ In NEL, although microbial pathogens differ from
SLE, it is similar to the overexpression of TLR7, with abundant IFN- $\alpha$ production (Fig. 5).

Future studies are required to elucidate the underlying regulatory mechanisms, and roles of TLR7 and TLR9 in the complex pathogenesis of NEL.

In conclusion, SLE is an autoimmune disease, whereas NEL is a systemic non-autoimmune disorder. The influence of TLR7 may be greater than that of TLR9 on the production of IFN- $\alpha$ in NEL. Although the pathogenesis of NEL is unclear at present, this study moved us one step closer to clarifying the pathogenesis.

When diagnosing NEL, it should be noted that the clinical course of the disorder is markedly diverse and a variety of treatments may be administered before biopsy of the lymph nodes. Therefore, the pathogenesis of NEL should be examined in detail taking these points into consideration.

\section{CONFLICT OF INTEREST}

The authors have no conflicts of interest, financial or otherwise, in relation to this study.

\section{REFERENCES}

1 Asano S, Akaike Y, Muramatsu T, et al. Necrotizing lymphadenitis: A clinicopathological and immunohistochemical study of four familial cases and five recurrent cases. Virchows Arch A Pathol Anat Histopathol. 1991; 418 : 215-223.

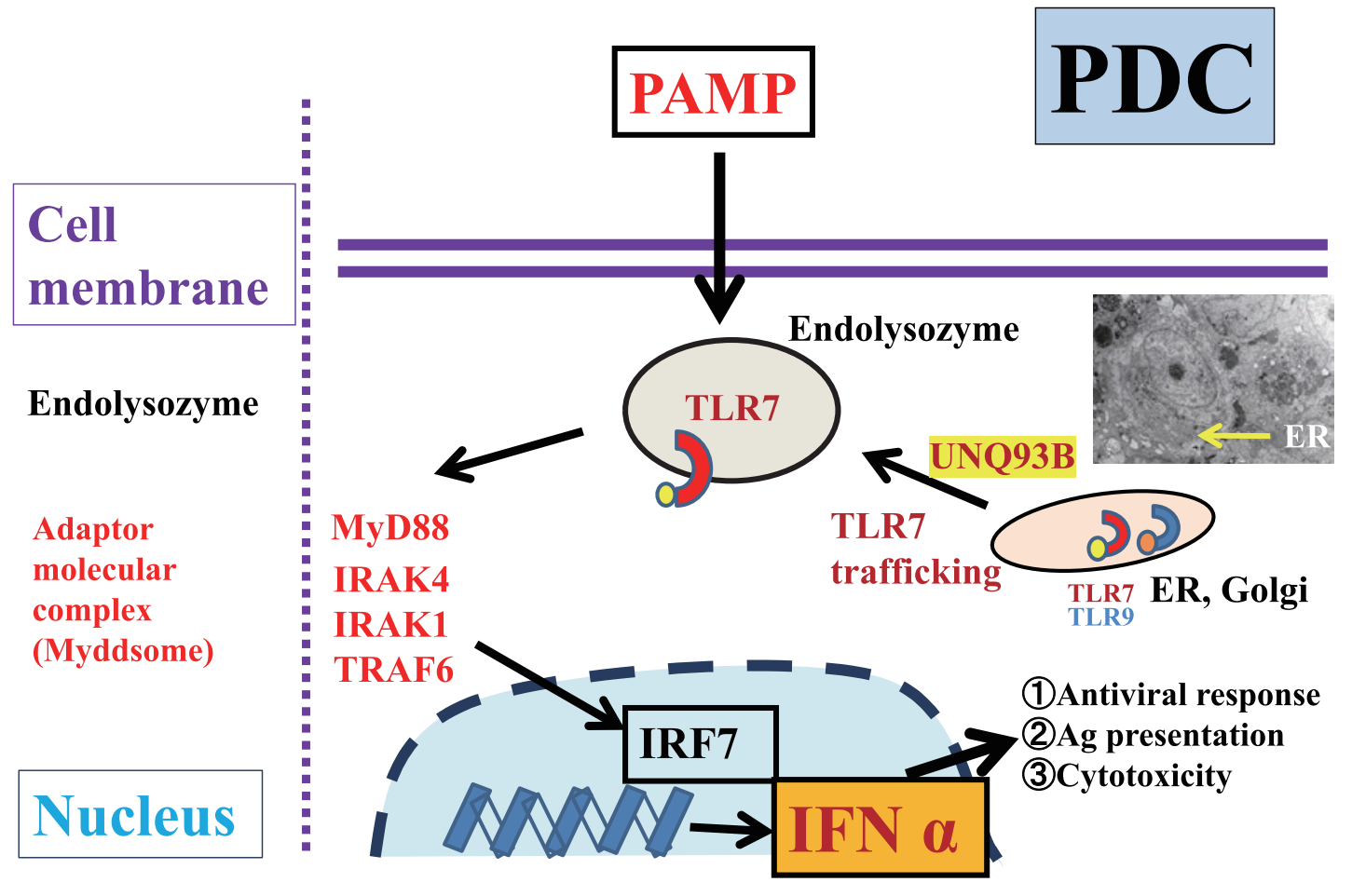

Fig. 4. Representative signaling pathways of TLR7 and TLR9 in PDCs in NEL. TLR7 and TLR9 present in the ER of PDCs under normal conditions, but when pathogens invade the host, TLR7 and TLR9 migrate to endolysosomes and recognize them quickly. Overexpression of TRL7 recruits adaptor molecular complexes such as MyD88 and other proteins. Later, it induces the production of IFN- $\alpha$. This signaling pathway is important for host defense against pathogens such as viruses. *PAMP; pathogen-associated molecular pattern. TLR7(9); Tolllike receptor 7(9). 


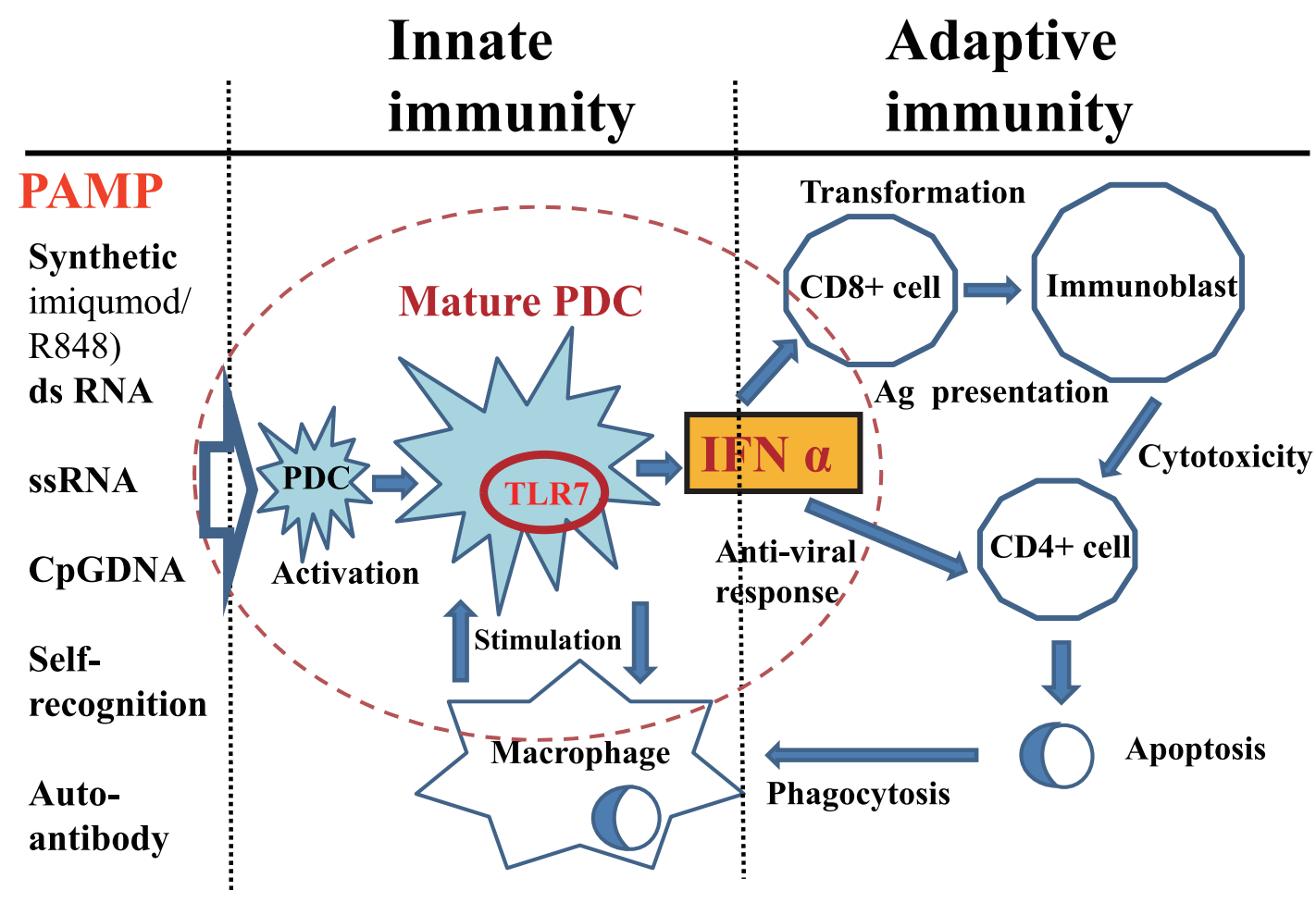

Fig. 5. Innate and adaptive immunity in NEL. Larger matured PDCs in the lesions indicate that the PDCs were activated by pathogenic stimulation. TLR7 in PDCs of NEL link the innate immunity and adaptive immunity such as antiviral response (IFN- $\alpha$ ), antigen presentation (blastic transformation of CD $8^{+}$cells), and cytotoxicity (apoptosis of $\mathrm{CD}^{+}$cells). *PAMP; pathogen-associated molecular pattern. TLR7; Toll-like receptor 7.

2 Asano S, Kanno H, Tominaga K, et al. Necrotizing lymphadenitis. Electron microscopical and immunohistochemical study. Acta Pathol Jpn. 1987; 37 : 1071-1084.

3 Pileri S, Kikuchi M, Helbron D, Lennert K. Histiocytic necrotizing lymphadenitis without granulocytic infiltration. Virchows Arch A Pathol Anat Histol. 1982; 395 : 257-271.

4 Turner RR, Martin J, Dorfman RF. Necrotizing lymphadenitis. A study of 30 cases. Am J Surg Pathol. 1983; 7 : 115-123.

5 Imamura $\mathrm{M}$, Ueno $\mathrm{H}$, Matsuura A, et al. An ultrastructural study of subacute necrotizing lymphadenitis. Am J Pathol. 1982; 107 : 292-299.

6 Takeshita M, Tashiro K, Mitsui T, et al. Activity of 2' $-5^{\prime}$ oligoadenylic acid synthesizing enzyme and histologic feature of histiocytic necrotizing lymphadenitis. J Clinic Hematol. 1986; $28: 370$ [in Japanese].

7 Sato H, Asano S, Mori K, et al. Plasmacytoid dendritic cells (PDCs) may produce interferon- $\alpha$ (IFN- $\alpha$ ) and play an important role for $\mathrm{CD}^{+}$cells and $\mathrm{CD} 8^{+}$cells in necrotizing lymphadenitis. J Clin Exp Hematop. 2015; 55 : 127-135.

8 Asano S, Mori K, Yamazaki K, et al. Necrotizing lymphadenitis (NEL) is a systemic disease characterized by blastic transformation of CD8+ cells and apoptosis of CD4+ cells. Virchows Arch. 2014; $464: 95-103$

9 Pilichowska ME, Pinkus JL, Pinkus GS. Histiocytic necrotizing lymphadenitis (Kikuchi-Fujimoto disease): lesional cells exhibit an immature dendritic cell phenotype. Am J Clin Pathol. 2009; $131: 174-182$.

10 Kishimoto K, Tate G, Kitamura T, Kojima M, Mitsuya T. Cytologic features and frequency of plasmacytoid dendritic cells in the lymph nodes of patients with histiocytic necrotizing lymphadenitis (Kikuchi-Fujimoto disease). Diagn Cytopathol. 2010; 38 : 521-526.

11 Grouard G, Rissoan MC, Filgueira L, et al. The enigmatic plasmacytoid T cells develop into dendritic cells with interleukin (IL)-3 and CD40-ligand. J Exp Med. 1997; 185 : 1101-1111.

12 Cella M, Jarrossay D, Facchetti F, et al. Plasmacytoid monocytes migrate to inflamed lymph nodes and produce large amounts of type I interferon. Nat Med. 1999; 5 : 919-923.

13 Olweus J, BitMansour A, Warnke R, et al. Dendritic cell ontogeny: A human dendritic cell lineage of myeloid origin. Proc Natl Acad Sci USA. 1997; 94 : 12551-12556.

14 Strobl H, Scheinecker C, Riedl E, et al. Identification of CD68 ${ }^{+}$lin- peripheral blood cells with dendritic precursor characteristics. J Immunol. 1998; 161 : 740-748.

15 Jahnsen FL, Lund-Johansen F, Dunne JF, et al. Experimentally induced recruitment of plasmacytoid (CD123high) dendritic cells in human nasal allergy. J Immunol. 2000; 165 : 4062-4068.

16 Tang F, Du Q, Liu YJ. Plasmacytoid dendritic cells in antiviral immunity and autoimmunity. Sci China Life Sci. 2010; 53 : 172-182.

17 Siegal FP, Kadowaki N, Shodell M, et al. The nature of the principal type 1 interferon-producing cells in human blood. Science. 1999; 284 : 1835-1837.

18 Svensson H, Johannisson A, Nikkilä T, Alm GV, Cederblad B. The cell surface phenotype of human natural interferon-alpha producing cells as determined by flow cytometry. Scand J Immunol. 1996; 44 : 164-172.

19 Svensson H, Cederblad B, Lindahl M, Alm G. Stimulation of 
natural interferon-alpha/beta-producing cells by Staphylococcus aureus. J Interferon Cytokine Res. 1996; 16 : 7-16.

20 Fitzgerald-Bocarsly P. Human natural interferon- $\alpha$ producing cells. Pharmacol Ther. 1993; $60: 39-62$.

21 Karrich JJ, Jachimowski LCM, Uittenbogaart CH, Blom B. The plasmacytoid dendritic cell as the Swiss army knife of the immune system: molecular regulation of its multifaceted functions. J Immunol. 2014; $193: 5772-5778$.

22 Fonteneau JF, Gilliet M, Larsson M, et al. Activation of influenza virus-specific $\mathrm{CD}^{+}$and $\mathrm{CD}^{+} \mathrm{T}$ cells: a new role for plasmacytoid dendritic cells in adaptive immunity. Blood. 2003; $101: 3520-3526$.

23 Di Pucchio T, Chatterjee B, Smed-Sörensen A, et al. Direct proteasome-independent cross-presentation of viral antigen by plasmacytoid dendritic cells on major histocompatibility complex class I. Nat Immunol. 2008; 9 : 551-557.

24 Guillerme JB, Boisgerault N, Roulois D, et al. Measles virus vaccine-infected tumor cells induce tumor antigen cross-presentation by human plasmacytoid dendritic cells. Clin Cancer Res. 2013; $19: 1147-1158$.

25 Tel J, Schreibelt G, Sittig SP, et al. Human plasmacytoid dendritic cells efficiently cross-present exogenous Ags to $\mathrm{CD}^{+} \mathrm{T}$ cells despite lower Ag uptake than myeloid dendritic cell subsets. Blood. 2013; $121:$ 459-467.

26 Farkas L, Beiske K, Lund-Johansen F, Brandtzaeg P, Jahnsen FL. Plasmacytoid dendritic cells (natural interferon- $\alpha /$ $\beta$-producing cells) accumulate in cutaneous lupus erythematosus lesions. Am J Pathol. 2001; 159 : 237-243.

27 Anderson KV, Bokla L, Nüsslein-Volhard C. Establishment of dorsal-ventral polarity in the drosophila embryo: The induction of polarity by the Toll gene product. Cell. 1985; 42 : 791-798.

28 Lemaitre B, Nicolas E, Michaut L, Reichhart JM, Hoffmann JA. The dorsoventral regulatory gene cassette spätzle/Toll/cactus controls the potent antifungal response in Drosophila adults. Cell. 1996; $86:$ 973-983.

29 Akira S, Uematsu S, Takeuchi O. Pathogen recognition and innate immunity. Cell. 2006; 124 : 783-801.

30 Medzhitov R, Janeway CA Jr. Innate immunity: the virtues of a nonclonal system of recognition. Cell. 1997; 91 : 295-298.

31 Rock FL, Hardiman G, Timans JC, Kastelein RA, Bazan JF. A family of human receptors structurally related to Drosophila Toll. Proc Natl Acad Sci USA. 1998; 95 : 588-593.

32 Kawai T, Akira $\mathrm{S}$. The role of pattern-recognition receptors in innate immunity: update on Toll-like receptors. Nat Immunol. 2010; $11: 373-384$

33 Celhar T, Magalhães R, Fairhurst AM. TLR7 and TLR9 in SLE: when sensing self goes wrong. Immunol Res. 2012; 53 : 58-77.

34 Blasius AL, Beutler B. Intracellular toll-like receptors. Immunity. 2010; $32: 305-315$.

35 Lee SMY, Kok KH, Jaume M, et al. Toll-like receptor 10 is involved in induction of innate immune responses to influenza virus infection. Proc Natl Acad Sci USA. 2014; 111 : 3793-3798.
36 Regan T, Nally K, Carmody R, et al. Identification of TLR10 as a key mediator of the inflammatory response to Listeria monocytogenes in intestinal epithelial cells and macrophages. J Immunol. 2013; $191:$ 6084-6092.

37 Zhang SY, Jouanguy E, Ugolini S, et al. TLR3 deficiency in patients with herpes simplex encephalitis. Science. 2007; 317 : 1522-1527.

38 Bernard JJ, Cowing-Zitron C, Nakatsuji T, et al. Ultraviolet radiation damages self noncoding RNA and is detected by TLR3. Nat Med. 2012; 18 : 1286-1290.

39 Takemura N, Kawasaki T, Kunisawa J, et al. Blockade of TLR3 protects mice from lethal radiation-induced gastrointestinal syndrome. Nat Commun. 2014; $5: 3492$.

40 Diebold SS, Kaisho T, Hemmi H, Akira S, Reis e Sousa C. Innate antiviral responses by means of TLR7-mediated recognition of single-stranded RNA. Science. 2004; 303 : 1529-1531.

41 Heil F, Hemmi H, Hochrein H, et al. Species-specific recognition of single-stranded RNA via toll-like receptor 7 and 8 . Science. 2004; $303:$ 1526-1529.

42 Lund JM, Alexopoulou L, Sato A, et al. Recognition of singlestranded RNA viruses by Toll-like receptor 7. Proc Natl Acad Sci USA. 2004; 101 : 5598-5603.

43 Bauer S, Pigisch S, Hangel D, Kaufmann A, Hamm S. Recognition of nucleic acid and nucleic acid analogs by Tolllike receptors 7, 8 and 9. Immunobiology. 2008; $213: 315-328$.

44 Mancuso G, Gambuzza M, Midiri A, et al. Bacterial recognition by TLR 7 in the lysosomes of conventional dendritic cells. Nat Immunol. 2009; $10:$ 587-594.

45 Coban C, Igari Y, Yagi M, et al. Immunogenicity of whole-parasite vaccines against Plasmodium falciparum involves malarial hemozoin and host TLR9. Cell Host Microbe. 2010; 7 : 50-61.

46 Kawasaki T, Kawai T. Toll-like receptor signaling pathways. Front Immunol. 2014; 5 : 461-467.

47 Casrouge A, Zhang SY, Eidenschenk C, et al. Herpes simplex virus encephalitis in human UNC-93B deficiency. Science. 2006; $314: 308-312$.

48 Fukui R, Saitoh S, Kanno A, et al. Unc93B1 restricts systemic lethal inflammation by orchestrating Toll-like receptor 7 and 9 trafficking. Immunity. 2011; $35: 69-81$.

49 Gilliet M, Cao W, Liu YJ. Plasmacytoid dendritic cells: sensing nucleic acids in viral infection and autoimmune diseases. Nat Rev Immunol. 2008; 8 : 594-606.

50 Kono H, Rock KL. How dying cells alert the immune system to danger. Nat Rev Immunol. 2008; 8 : 279-289.

*The summary of this study was presented at the $31^{\text {th }}$ European Congress of Pathology on September 7-11, 2019 in Nice. 